\title{
¿ES LA PEDAGOGÍA LA HERRAMIENTA DE LA PRÁCTICA DOCENTE? EL CASO DE LA EVALUACIÓN
}
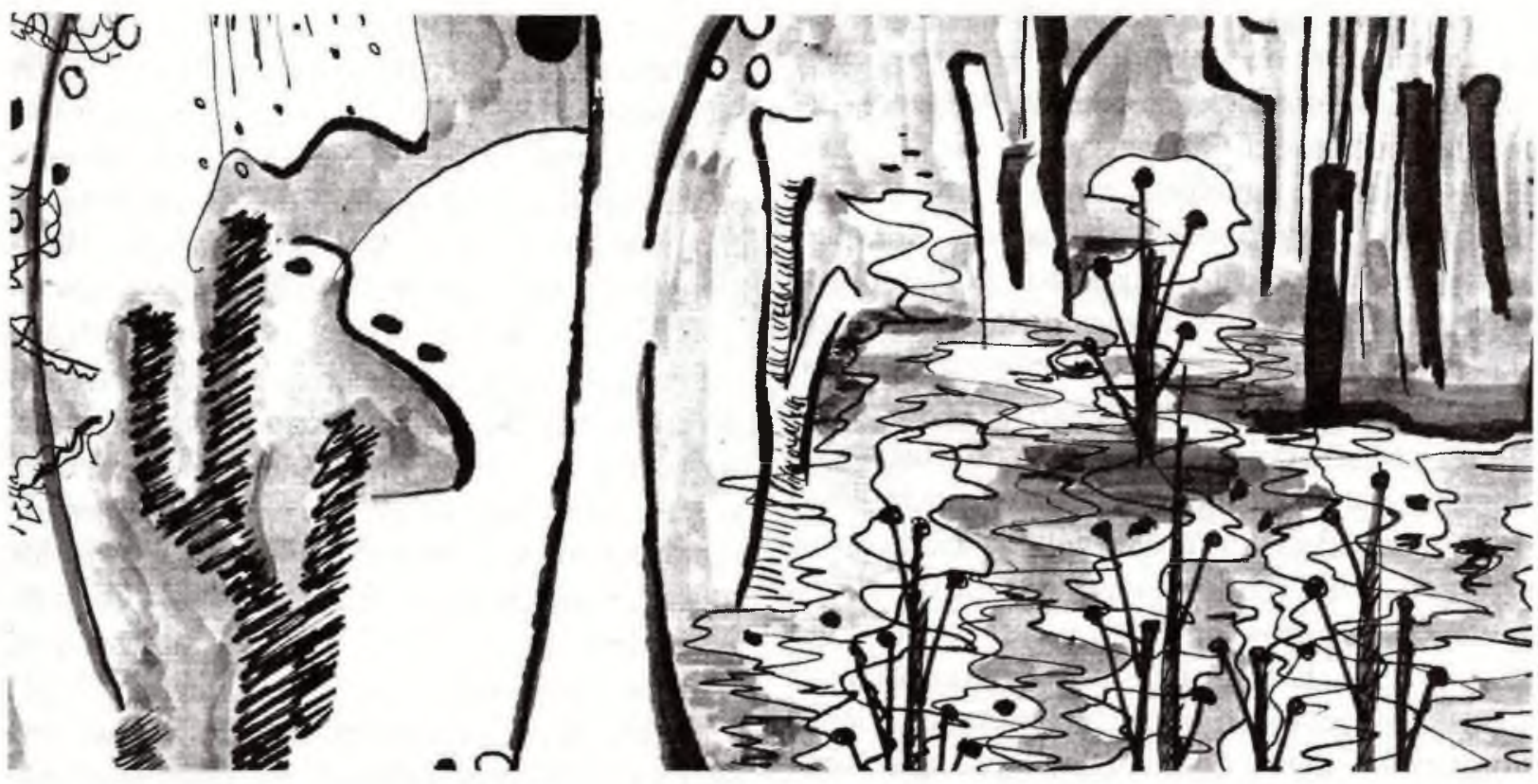

LICET KATERINE SUÁREZ ESTUDIANTE PROGRAMA DE LENGUA CASTELLANA FACULTAD DE EDUCACIÓN katercor427@yahoo.com.mx

"El quehacer educativo -ya sea como "receptores" o como formadores- obliga a reflexionar acerca del lugar que deseamos ocupar dentro del proceso de formación permanente en el cual estamos insertos"

GABRIELA MESSINA

\section{RESUMEN}

El presente artículo busca mostrar la importancia de la integración de los saberes disciplinario y pedagógico en uno de los mecanismos de la práctica docente y al cual se le ha dado poca importancia en la propia práctica de la universidad: la evaluación. Se hace una reflexión discursiva desde la óptica del discente, ilustrando con casos de la realidad del programa de lengua castellana.

\section{PALABRAS CLAVE}

saber disciplinar, saber pedagógico, evaluación, profesor, estudiante.

\section{ABSTRACT}

This article intends to show how important it is to combine to types of knowledge (pedagogical and disciplinary) in the assessment. This is a very important mechanism in the practice of teaching that has been put aside when it comes to College life. This article also shows some clear examples taken from the USCO's Program for Spanish Language from student's point of view.

\section{KEY WORDS}

disciplinaryknowledge, pedagogical knowledge, assessment, teacher, student 
$\mathrm{E}$ proceso de formación de un especialista en docencia ${ }^{18}$, lleva consigo la posibilidad de integrar el saber pedagógico y el saber disciplinar. Es una verdad innegable. Para el presente texto, deseamos adentrarnos en el papel que debería jugar la pedagogía, (entendida inicialmente como teoría y práctica de la educación ${ }^{19}$ ) en la optimización del ejercicio docente. No se trata entonces de reproducir ${ }^{20}$ y mecanizar una extensa terminología que se desprende del ámbito pedagógico en una licenciatura como es la de lengua castellana, cuyas asignaturas del denominado componente de facultad, a saber, pedagogía, psicología, currículo, epistemología de la educación y metodología de la investigación que intentan contribuir con la configuración de un docente integro en pro del desarrollo regional (ver Visión y Misión del Programa), sino de integrar los diferentes saberes (conocimientos) para poder llevar a cabo una adecuada 'evaluación' de los procesos de enseñanza/aprendizaje en el ejercicio de nuestra profesión: Ser profesores de una especialidad (en nuestro caso, de lengua castellana), consolidando un marco teórico que en cierta medida garantice la 'formación integral' de una comunidad estudiantil y que a la par den crédito de la interdisciplinariedad académica brindada en la universidad como espacio académico que desarrolla el pensamiento científico. Somos inicialmente estudiantes que aprendemos y somos guiados para luego devenir en licenciados y convertirnos en guías.

Partimos del supuesto de que el proceso enseñanza/aprendizaje en una carrera de licenciatura debe permitirle al estudiante ${ }^{21}$, "aprender no sólo a ser, a convivir, a hacer, a pensar desde el énfasis disciplinar sino también, aprender a enseñar dichos aprendizajes" ${ }^{22}$. En esa perspectiva, la evaluación debe apoyarse en tres elementos: el contenido de los procesos de aprendizaje; la naturaleza de los procesos de aprender a enseñar -e implícitamente aprender a aprender- y las características de los entornos de aprendizaje $^{23}$. Ese sería el ideal, no obstante, el caso más recurrente de evaluación es el llevado a cabo por la mayoría de profesores cuando intentan medir qué han aprendido los estudiantes en su respectiva asignatura (para el caso de la licenciatura en lengua castellana, unos contenidos disciplinares de los cursos del componente específico), ignorando, a mi modo de ver, los procesos cognitivos subyacentes. Además, no quiero afirmar que hay una mala intención en esa evaluación, lo que sí hay es una ignorancia en cómo evaluar adecuadamente, que termina siendo nociva para nuestra formación ${ }^{24}$. La evaluación resulta ser tan solo una actividad de control escolar, en la mayoría de los casos

18 Que en nuestro medio colombiano hemos denominado "licenciados" en algún campo específico del saber. Tenemos pues licenciados en lengua castellana, en matemáticas, en filosofía, en ciencias sociales, en ciencias naturales, hasta licenciados en psicología y pedagogía (programa ofrecido por la Universidad Pedagógica Nacional). El licenciado (profesor) es el especialista formado en las facultades de educación y el más indicado para la conciliación de los saberes disciplinar y pedagógico.

19 Para comprender cómo se ha llegado a la definición de la pedagogía, recomendamos la lectura de los siguientes libros: Introducción a la pedagogía general (Franco Frabboni y Franca Pinto, 2006), Filosofía, Pedagogía y tecnología (Germán Vargas Guillén, 2006), Tratado de pedagogía (Immanuel Kant, 2004) y Psicología y Pedagogía (Jean Piaget, 1969).

20 Reproducción en términos de Bourdieu. Consúltense dos de sus libros más conocidos y pertinentes para esta temática: La Reproducción. Elementos para una teoría del sistema de enseñanza, en coautoría con J.C. Passeron, 2002 y ¿Qué significa hablar? Economía de los intercambios lingüisticos, 2001.

21 Se supone que el individuo que juega el rol de profesor ya lo ha logrado.

22 Gómez Esteban, 1999, 37.

23 Op. Cit página 38. En otras palabras, estos tres elementos son los que nos ayudarían a responder una pregunta planteada desde comienzos de 2008 y que empezó a responderse formalmente en el Seminario sobre Sistematización organizado por PACA: "¿Qué tipo de variables se deben tener en cuenta para evaluar de manera justa a un estudiante del programa de lengua castellana?". En nuestra experiencia estudiantil, observamos que en la evaluación de nuestro desempeño se tiene en cuenta tan solo el primer elemento.

$24 \mathrm{El}$ profesor Nelson López, 2006, 139 lo ilustra bastante bien: “La Evaluación entendida como el proceso académico mediante el cual se constata el logro de avances y desarrollos formativos, resultados de los procesos curriculares y de la naturaleza de las prácticas pedagógicas de formación, y a la vez, factor de revisión y modificación de los mismos, se constituye en un proceso esencial en la constitución de identidades profesionales. No obstante, hoy se encuentra significativamente influenciada por una visión procedimental que la reduce la mayoría de las veces a un acto de mera y simple calificación, en lo que a la evaluación interna hace referencia." (El destacado es nuestro). Y esto no es todo; lograr ser docente no significa que sus procesos cognitivos hayan sido los más destacados (en términos de cultivados): "Los mismos docentes han recibido en muchos casos una formación muy deficiente y reproducen en sus interacciones pedagógicas las fallas de comprensión de conceptos y de manejo de lenguajes que los han marginado a ellos mismos y a sus maestros de conocimiento." Carlos A. Hernández, 2001,26. 
cuantitativa, en donde los hábitos tradicionalistas, por no decir arcaicos, se reflejan en un conjunto de normas cerradas que en la mayoría de los casos no le dan 'autonomía'25 al estudiante. Por ejemplo se le da una importancia exagerada al llamado a lista (verificar asistencia al comienzo y al final del curso), al uso permanente del video beam en las exposiciones, a la entrega de recordatorios, a la dramatización de obras literarias, al carnaval de disfraces, a la asistencia obligatoria a las asesorías previa actividad de exposición, al relato textual de lo que dice el autor de un texto dejando de lado la opinión personal, a la repetición de las glosas de los maestros, a la repetición de los fragmentos de obras, al trabajo con los textos que son preferidos por los docentes ${ }^{26}$ etc. ¿Por qué criticamos estas actividades? Las criticamos precisamente porque son actividades polarizadas, fruto de la opinión personal (subjetiva) de un docente que defiende una (im)postura sin consultarla con los demás miembros de la comunidad del programa. Las criticamos porque no están conectadas explícitamente con el proceso enseñanza/ aprendizaje del área específica. Tal y como se mencionan en su aislamiento, la evaluación de dichas actividades no logra trascender la simple valoración de logros frente a objetivos planteados. $Y$ es aquí en donde retomamos la necesidad de integrar lo disciplinar y lo pedagógico. El proceso de aprendizaje involucra tanto al maestro como al estudiante. Los dos reconfiguran un objeto de estudio y lo abordan desde una óptica discursiva. Siguiendo a Adriana Bolívar 2007, el 'discurso' tiene dos dimensiones. Una es la interacción social ${ }^{27}$ y la otra es la cognición. Ambas están presentes en la actividad evaluativa en la Universidad. Sin embargo, para nuestro propósito, la segunda es la que serviría como puente para integrar los saberes que tratamos. En palabras de A. Bolívar, "el discurso es cognición porque las personas construyen su conocimiento del mundo y adaptan sus representaciones a los contextos en los que viven según las opciones y/o limitaciones que se les ofrezcan." ${ }^{28}$

Es alarmante además que un docente universitario deje de lado la incidencia de la sistematización ${ }^{29}$, (entendida ésta como la reconfiguración de nuevos sentidos que apuntan a considerar la integración social, la interacción, el intercambio semiótico en la labor investigativa de una manera explícita), en la consolidación de un marco teórico de evaluación para darle importancia exagerada a los procesos de repetición catedrática, como "validez" del conocimiento adquirido en clase. Podemos plantear un nuevo interrogante: iel docente universitario maneja adecuadamente la herramienta pedagógica en la enseñanza de su cátedra?, es decir, ¿construye un esquema teórico/metodológico que conduzca al estudiante a redescubrir sus procesos de cognición? No olvidemos el valor de las variables culturales $y$ sociales en el proceso de enseñanza/aprendizaje de los actores educativos. Así entonces, si el docente universitario comprende tales variables, asumiendo de manera profesional la validez de la teoría del aprendizaje propuesta por Vygotsky,

25 Autonomía ideal y anhelada por el estudiante universitario y que remite sin duda alguna al concepto de 'mayoría de edad' kantiano.

26 Citando el caso de lengua castellana, en el área de literatura hay docentes que consideran ciertas obras literarias como lo máximo, y prescriben otras por no estar al nivel de las primeras. Comparan, sin fórmula de juicio, El Quijote de la Mancha con Rosario Tijeras (¿Por qué semejante comparación?). Critican la producción de un autor como Jairo Aníbal Niño por considerarla facilista y carente de profundidad. Atacan y ridiculizan en clase a aquellos estudiantes que no han leído La Vorágine o Tierra de promisión. Se les olvida que no todos los estudiantes de la USCO son originarios del Huila. Defienden algunos los clásicos griegos como lo máximo pero sin dar mayores razones. Para el caso de la lingüística no hay mucha diferencia. Se recomiendan en varios de los cursos la lectura de manuales pero se desaconseja la lectura de las propias fuentes. Los textos de Saussure, Chomsky, Lyons, Halliday, Benveniste no son tratados, ni siquiera el propio libro de uno de los profesores del área. Y como si esto fuera poco, se quiere hacer creer que la cultura agustiniana es el non plus ultra de la creación humana. Es tan solo una muestra entre otras tantas que igualmente son imponentes. Es todo.

27 Que podemos relacionarla con lo propuesto por el profesor Leonel Arias (1993), en lo tocante al respeto y a la dignidad humanas: ".... la dignidad de un ser vivo o de una especie de seres vivos consiste en el atributo o conjunto de atributos que lo hacen merecedor de especiales consideraciones en la forma de tratar."

$282007,22$.

29 Al respecto afirma Ghiso: "La formación en sistematización, como propuesta crítica generadora de conocimiento, exige una deconstrucción epistemológica de los presupuestos que fundamentan la investigación científica tradicional, entre otras cosas, porque su objeto de estudio es la práctica social, profesional, académica o formativa." (Falta cotejo).

30 Lev Vygotsky, 1995, sin dejar de lado por supuesto las tesis de Jerome Bruner, Jean Piaget, Humberto Maturana entre otros tantos. 
ya no se seguirían esquemas tradicionalistas que transforman el conocimiento en imposturas ${ }^{31}$ académicas, sino se lograría empalmar los saberes objeto de nuestro estudio.

Ahora bien, si bien es cierto que venimos hablando del método de evaluación que se lleva a cabo en la universidad -que no es el óptimo dentro del proceso de sistematización- debemos tener en cuenta que algunos profesores de nuestro programa están tratando de proponer actividades académicas que logren la integración de los saberes disciplinar y pedagógico. ¿Qué se percibe de este intento? En primer lugar, han logrado aclararnos que nuestra formación apunta a preparar un docente. Esto implica que debe manejar un saber pedagógico, debe comprender cómo funciona el cerebro de los estudiantes en los procesos de aprendizaje ${ }^{32}$, debe ser capaz de concebir y poner a funcionar modelos pedagógicos que favorezcan los procesos de aprendizaje, debe ser un cultivador de valores, de respeto y de tolerancia. De igual forma, debe manejar un saber disciplinar que en lengua castellana consiste en trabajar los principios de la teoría lingüística y su correlato la lingüística aplicada a la enseñanza de la lengua materna. Para el caso de literatura, el trabajo con la teoría literaria y el análisis global de las obras. En segundo lugar, se persigue niveles de excelencia y búsqueda de integración con comunidades académicas nacionales e internacionales con el fin de proyectar la función del programa y de la Facultad. El licenciado en lengua castellana sabrá manejar un esquema pedagógico que incite al estudiante a consolidar la actividad de escritura y re- lectura (i.e. lectura inferencial y argumentativa), logrando así el desarrollo de criterios epistemológicos que conduzcan al estudiante a la configuración de un objeto de estudio ${ }^{33}$ que le permita llevar a cabo la actividad de investigación - que en últimas es uno de los objetivos de la educación superior-. $Y$ ya que nos adentramos en el tópico de la investigación, podemos preguntar ¿los profesores universitarios tienen definido su objeto de estudio? Es mas, ¿llevan a cabo una constante actividad de investigación? ¿La transmiten y vivencian en sus cátedras? ¿La investigación, como miembros de una Facultad de Educación, integra saber disciplinar y saber pedagógico? Dichos interrogantes deben ser respondidos apoyándonos en una base epistemológica cuyo campo desemboque en una actividad científica. Sin embargo, es evidente que muchos docentes de nuestra institución universitaria y más específicamente de nuestro programa de Lengua Castellana, no tienen claridad, tanto en la formulación y tratamiento de los criterios epistemológicos de cada uno de sus campos académicos especializados, como de la actividad de investigación que algunos manejan. Esto se puede constatar en los propios procesos de transmisión de contenidos y en la respectiva evaluación que llevan a cabo de ellos. Algunos de los cursos de literatura así como los de lingüística y didáctica se han convertido en prueba fehaciente de ello. Si tomamos la faceta del discurso como interacción social, podemos darnos cuenta de que en los propios intercambios entre los estudiantes, el descontento frente a la presentación y evaluación de cursos es pan cotidiano. No hay suficiente intercambios académicos fuera del aula de clase ${ }^{34}$ ¿Por qué no se crean espacios de intercambio de experiencias, de vivencias, de lecturas ${ }^{35}$, con

31 Seguimos a Nelson López: "Sobre el concepto 'impostura' se encuentran las más variadas concepciones y definiciones, sin embargo, en el marco de este artículo se conciben como 'tratamientos y actitudes que rinen con el rigor, la seriedad y la argumentación que caracteriza el accionar académico, investigativo y científico, que repercuten en los niveles de calidad y excelencia debilitando los procesos de pertenencia social y pertinencia académica de la educación como proceso formativo'. Estas imposturas ubicadas en el campo educativo son portadoras de poder y control y, por lo tanto se convierten en mecanismos reguladores y legitimadores de formas específicas de subjetividad (identidad profesional), como también, en dispositivos de reproducción cultural." $(2009,26)$

32 "El conocimiento de cómo aprende el cerebro podría tener, y tendrá, un gran impacto en la educación. Comprender los mecanismos cerebrales que subyacen al aprendizaje y la memoria, así como los efectos de la genética, el entorno, la emoción y la edad en el aprendizaje, podrían transformar las estrategias educativas y permitirnos idear programas que optimizarán el aprendizaje de personas de todas las edades y con las más diversas necesidades. Sólo comprendiendo cómo el cerebro adquiere y conserva información y destrezas seremos capaces de alcanzar los límites de su capacidad para aprender." Blakemore y Frith, 2008, 19. Esta temática ha sido tratada adecuadamente en el curso Lenguaje y Evolución Humana.

33 Entendido como una porción de la realidad cotidiana que se somete a la problematización y la formalización. En lengua castellana, podemos citar dos objetos claros: la comunicación y la significación.

34 No desconocemos por supuesto el esfuerzo para crear los semilleros de investigación. Sin embargo, estos también reproducen los puntos negativos que hemos tratado.

35 ¿Por qué no se ha trabajado, y no importa el espacio académico en donde se haga, un texto clásico e indispensable como lo es el "Enseñar no es transferir conocimiento" de Paulo Freire? 
el fin de hacer coevaluación del trabajo en el programa?

La pedagogía y la investigación (disciplinarpedagógica) se han desenfocado en una gran medida, ya que no hay un interés ni por parte del docente universitario, jugando este el papel de guía, ni por parte del estudiante como protagonista principal en cuanto a la realización de una propuesta de investigación. El papel del estudiante se ha vinculado al hábito de la memorización, la repetición y el plagio de conocimiento, ya que en muchos cursos los profesores se vanaglorian de los trabajos o parciales entregados por parte de los estudiantes como "respuesta exacta" a lo que ellos "enseñaron".

La educación superior no es sinónimo de almacenamiento de conceptos, la memorización de teorías, la repetición de criterios tradicionalistas; la educación superior es la vinculación a la actividad investigativa, en donde al profesional se le abren posibilidades de proponer un discurso académico sustentado en una constante actividad de reflexión científica y auto-cuestionamiento desde una perspectiva epistemológica; en donde sus saberes se incluyan en el trabajo investigativo, configurando de tal modo el enfoque pedagógico con el disciplinar. Hacia allá debemos caminar y ese debería ser uno de los propósitos de la comunidad académica de nuestra Facultad de Educación.

\section{BIBLIOGRAFÍA}

ARIAS, L. (1993). Ideas para una axiología biocéntrica en las relaciones humanas. Documento inédito.

BLAKEMORE, S-J. \& FRITH, U. (2008) Cómo aprende el cerebro. Las claves para la educación. Barcelona: Ariel.

BOLIVAR, A. (comp.) (2007). Análisis del discurso. ¿Por qué y para qué? Caracas: Universidad Central de Venezuela y Los libros de El Nacional.

BOURDIEU. P. (2001). ¿Qué significa hablar? Economía de los intercambios lingüísticos. Madrid: Akal.

BOURDIEU, P. \& PASSERON, J-C. (2002). La reproducción. Madrid: Editorial Popular.
FRABBONI, F. \& FRANCA, M. (2006). Introducción a la Pedagogía General. México: Siglo XXI Editores.

FREIRE, P. (1997) Enseñar no es transferir conocimiento. En Pedagogía de la autonomía. México: Siglo XXI Editores.

GOMEZ ESTEBAN, J.H. (1999). Relaciones entre saber pedagógico y saber disciplinar. En Acreditación previa. Compromiso institucional. Bogotá: Universidad Distrital.

HERNANDEZ, C.A. (2001). Aproximación a un estado del arte de la enseñanza de las ciencias en Colombia. En M. HENAO Y J.O. CASTRO (comp.) Estado del Arte de la Investigación en Educación y Pedagogía en Colombia, tomo II. Bogotá: Icfes, Colciencias y Sociedad Colombiana de Pedagogía.

KANT, I. (2004). Tratado de Pedagogía. Cali: Universidad del Valle.

LOPEZ, N. (2006). Acerca de la Cultura de la Evaluación en la Universidad Surcolombiana: Una aproximación crítica desde la experiencia ECAES. En GRUPO PACA, Evaluación por Competencias: Un reto por enfrentar y un proceso por construir. Neiva: Universidad Surcolombiana.

LOPEZ, N. (2009). La investigación como estrategia de debilitamiento de las denominadas "imposturas académicas". En Revista PACA. Neiva: Universidad Surcolombiana.

PIAGET, J. (1969). Psicología y Pedagogía. Barcelona: Ariel.

VARGAS GUILLEN, G. (2006). Filosofía, Pedagogía y Tecnología. Bogotá: Universidad Pedagógica Nacional, Ediciones San Pablo.

VYGOTSKY, L. (1995). Pensamiento y lenguaje. Barcelona: Paidós 